\title{
Peer Group: A New Approach of Nursing Intervention
}

\author{
${ }^{1,2}$ Suyanto*, ${ }^{3}$ Moses Glorino Rumambo Pandin \\ ${ }^{1}$ Faculty of Nursing, Univesitas Islam Sultan Agung, Semarang, Indonesia \\ ${ }^{2}$ Doctoral Program of Nursing Faculty, Universitas Airlangga Surabaya, Indonesia \\ ${ }^{3}$ Department of English Literature, Faculty of Humanities, Universitas Airlangga, Indonesia \\ *Corespoding Author \\ Email: suyanto@unissula.ac.id
}

\begin{abstract}
Received:
Revised:

Accepted:

Published:

29 June 2021

2 July 2021

27 July 2020

31 July 2021

Abstract

The development of nursing, especially related to the nursing intervention approach, is running so fast. This can be seen from the use of peer group support in nursing interventions in individual humans. The purpose of this literature is to find the impact of implementing nursing interventions using a peer group support approach. This literature review method uses JBI and Prisma on 120 articles taken from journal databases, namely Scopus, PubMed and ScienceDirect. From the articles analyzed, it was found that the application of peer groups can improve individual abilities both in psychological and behavioral aspects. The application of the peer group approach is able to be one of the approaches in the world of nursing in carrying out nursing actions today.
\end{abstract}

Keyword: Peer group support; peer group education and technology.

\section{INTRODUCTION}

The nursing paradigm consists of health, nursing, environment and human. One of the main components in the nursing paradigm is human. Humans are social creatures, where humans cannot live alone. Nurses with the role of care providers should re-realize the concept that humans are social creatures. In some cases where nurses have to provide intervention, it cannot be done only with an individual approach, but there is a need for a peer group support approach (Williams et al., 2019). One limitation of using an individual approach, especially in cases closely related to psychology, such as social isolation (Page-Reeves et al., 2021).

The limitations of the individual approach need other solutions that can be used by nurses in carrying out nursing actions. The peer group support approach can be one solution to these limitations. This is based on the fact that peer group support emphasizes togetherness among group members, especially seen from the same feelings towards what is being experienced (Toija et al., 2019).

The use of the peer group support approach can be used to improve individual behavior in the group. The peer group support approach can improve welfare, increase self-efficacy (Toija et al., 2019). Peer group education can also improve health, physical, emotional, and social wellbeing, reduce depression, and have a better future perspective (Page-Reeves et al., 2021). Various 
forms of peer group support are used, among others, the use of face-to-face peer groups, the use of technology, and the use of peer group education. The purpose of this literature review is to see the application of peer group support in improving aspects of psychology and patient behavior.

\section{METHODS}

The method used in this literature review uses the JBI and PRISMA protocol (Kamioka, 2019). Articles were obtained by writing keywords in 3 database journals, namely Scopus, Pubmed and Science Diret. The keywords included are peer group support, peer group education and technology peer group. The incluation criteria in this study are articles that appear selected from the year of publication, starting from 2019-2021, qualitative and quantitative research designs, and research areas in both hospitals and communities. Based on the PRISMA and JBI guidelines and inclusion criteria, 7 articles were obtained.

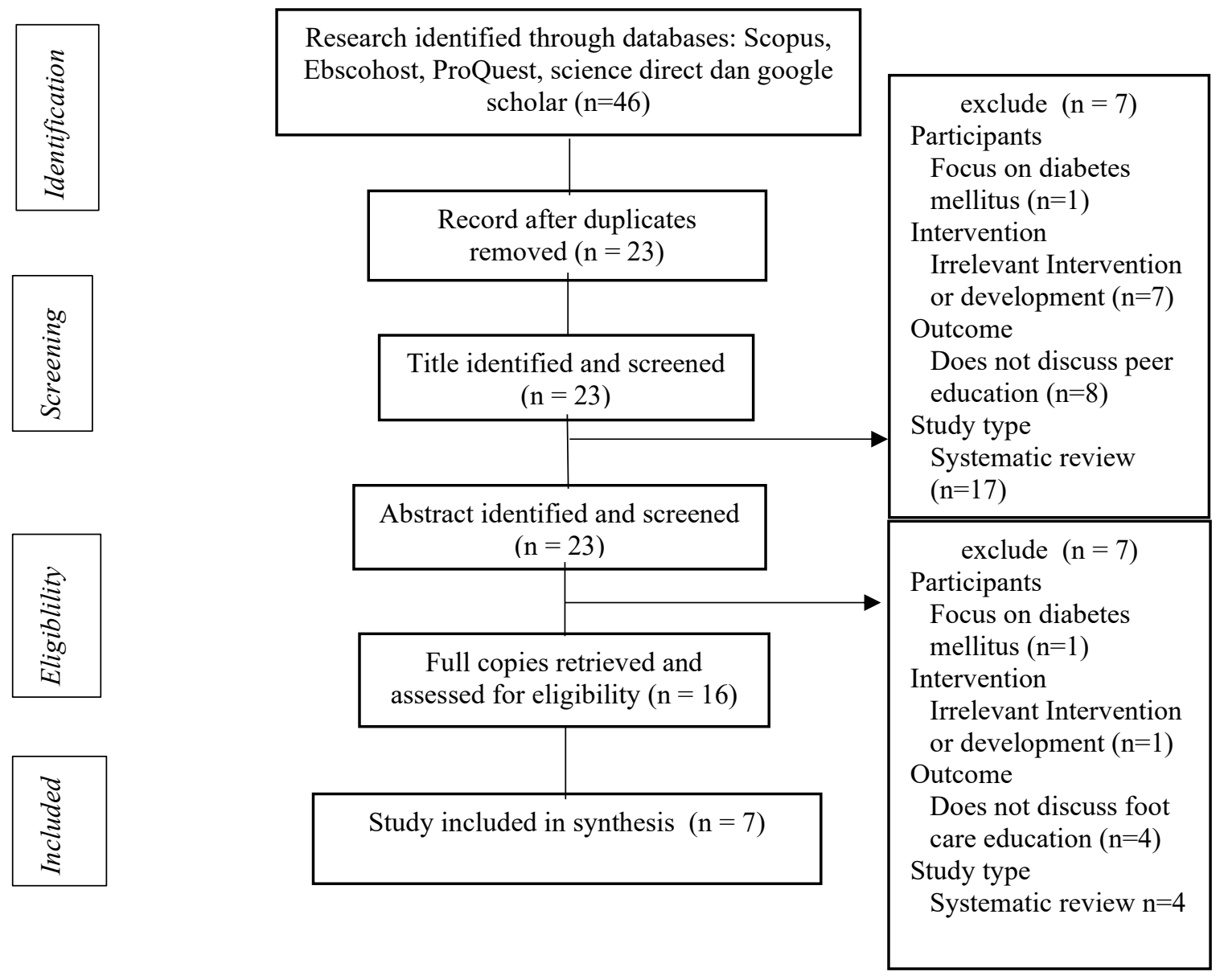

Figure 1. Preferred Reporting Items for Systematic Reviews and Meta Analysis (PRISMA) Flowchart 


\section{RESULTS}

The results of a review of 7 articles obtained from the journal database are as follows:

1. "Effectiveness of Technologically Enhanced Peer Support in Improving Glycemic Management Among Predominantly African American, Low-Income Adults with Diabetes".

Aims of research is to determine the impact of application of electronic health education combined with peer coaching. This study used a two-armed randomized controlled trial. The intervention was given for 6 months. The inclusion criteria included patients with diabetes mellitus who were hospitalized, HbA1C $8.0 \%$ with age less than 70 years. There was no difference in the increase in the value of $\mathrm{HbA1CThe} \mathrm{limitation} \mathrm{of} \mathrm{this} \mathrm{study} \mathrm{is} \mathrm{number} \mathrm{of} \mathrm{sample}$ and only one place. The conclusion of this study is that there is no difference between the use of e health education technology compared to peer coaching(Shannalee R. Martinez, Maresha S. Gay, 2016).

2. "Examining the effect of peer-support on self-stigma among persons living with HIV / AIDS"

This study discusses differences in stigma ratings of HIV / AIDS sufferers between peer support groups. This study used cross sectional study with inclusion criteria of HIV / AIDS patients. The results showed that peer groups can reduce stigma in HIV / AIDS patients even though it is not enough to reduce stigma (chime). The limitation of this research is that a facility-based study of PLHIV has been conducted previously, the data for PLHIV who are members of the support group community was not included in this study. Another limitation is about sample is not a clear representation of the small PLHIV population. This study shows that although peer groups have not been able to significantly reduce the stigma of society, having a peer group can increase the confidence of HIV / AIDS patients with the support of peer groups of HIV / AIDS patients (Chime et al., 2019).

3. "Peer-support: a coping strategy for nurses working at the Emergency Ambulance Service"

The Purpose of this research is about the experiences of nurses in using a peer supporting model. This research design is qualitative with the number of participants as many as 14 emergency room nurses. In order to prioritize prevention, the peer support model is deemed necessary in particular among peers and can be an adobt to be activated basiclly in situations considered to big emotional effect, just like pediatric patient management or suicide. The results of this study need peer support in reducing emotional stress in the emergency room (Carvello et al., 2019). This study is very interesting that nurses in the emergency room with work pressure that create emotional stress need peer support which is expected to reduce the emotional stress experienced by these nurses.

4. "Perceived social support and quality of life among adolescents in residential youth care: a cross-sectional study"

Adolescents who have experienced family problems, behavior problems in residential institutions need social support in improving their quality of life. This study focuses on the relationship between social support and adolescent quality of life. The study design was cross sectional with 400 adolescents in residential institutions with an age range of more than 18-23 years. The results 
show that there is a relationship between social support and adolescent quality of life. The limitations of this study include the cross-sectional design, variables that are more background to the respondents and instrument. This will provide more insight into the roles of different social support providers in improving the quality of life of adolescents. This study is important because adolescents who are in residential institutions need the importance of a lot of social support in their lives to improve their quality of life (Singstad et al., 2021).

5. "Those People Motivate and Inspire Me to Take My Treatment." Peer Support for Adolescents Living with HIV in Cape Town, South Africa"

This study examines the views of adolescents with HIV and caregivers regarding peer support. The study design was qualitative in 35 adolescents aged 12-19 years who received treatment and 35 caregivers. The results of this study found that there are benefits to having peer group support, especially in improving communication between adolescents with HIV. A weakness of this study is the possibility of bias in peer group participants where they may not want to criticize their friends who are in the same group (Rencken et al., 2021).

6. "Mobile Peer-Support for Opioid Use Disorders: Refinement of an Innovative Machine Learning Tool"

The use of information technology in implementing peer support needs to be developed. This study aims to create a peer group support-based platform for someone who experiences disorders due to drug use. 40 respondents were involved in this study. The results of this study are in the form of a platform which is the development of peer group support. In this research, we have not looked for the impact of this platform. This research is very good by combining information technology based on peer group support for someone who has a drug use disorder. In the future, there needs to be scientific evidence of the effectiveness of this platform (Scherzer et al., 2020).

7. "The outcomes of peer-led diabetes education in comparison to education delivered by health professionals in Iranian patients"

Patients with diabetes mellitus need to be educated. This study wants to compare the impact of education provided by health workers with peers on the self-care behavior of diabetes mellitus patients. The research design used clinical RCTs. A total of 40 respondents were included in this study, with incluation criteria consisting of age 40-65 years, people with type 2 diabetes, had a history of diabetes more than 6 months and were willing to participate in this study. Limitations of this study were among other participants selected from the same clinical setting and the possible interactions between participants. The study was carried out in urban areas of Iran, which limits the generalizability of the findings to non-urban areas of Iran. The results of this study found that peer education can improve the self-care behavior of diabetes mellitus patients. The limitation of this study is that because the respondents were taken from the same hospital, it was possible that there was interaction between these respondents (Ahmadi et al., 2018).

\section{DISCUSSION}

peer group is not an intervention, but peer group is an approach whose goal is to provide motivation, education and a community that supports each other (Warshaw \& Edelman, 2019). In its application, peer groups can be carried out into three approach activities, namely peer group 
support, peer group education and the use of peer group-based information technology. Peer group support is the most frequently used approach. The use of the peer group support approach tends to improve psychological and emotional aspects, although it cannot be separated from the behavioral aspects (Toija et al., 2019). Peer support can be done in the intensive care room where nurses can take this approach which is carried out on patients and families after leaving the incentive care room (McPeake et al., 2019). Peer support can also improve the quality of life in patients with lupus. This happens because social support from peer groups can increase understanding of self-care management for lupus (Williams et al., 2019). The development of a peer group exercise model can also improve mental and physical health in cancer patients (Adlard et al., 2019). The application of peer groups to mothers was also able to improve behavior to reduce obesity in their children (Cameron et al., 2019). The peer group support-based sister breastfeeding program provides proven resources for black women in urban communities in the south to deal with critical resources, including peer and breastfeeding support, and receiving essential pregnancy, breastfeeding, and postpartum education(Pyles et al., 2021) . The peer support approach for type 2 diabetes patients appears to be valued, as participants relate well to community health workers, who are ideally positioned in the health care system to provide services (Pienaar, 2021).Various research results regarding peer group support can be used as a reference that peer group support can be used in various types of diseases and patient conditions.

Peer group education can be applied to adolescents, the elderly, diabetes patients, and nurses (Backett-Milburn \& Wilson, 2000). Education using a peer group approach can improve self-care behavior in adolescents with asthma. For adolescents with peer led, they feel comfortable to have discussions with their peers. This is a factor that makes it easier for adolescents to apply self-care behavior (Richards et al., 2018). The application of peer led cancer with a peer support approach is also able to increase students' knowledge and self-esteem about cancer. This study used students' peers as group leaders in the cancer patient education process (Poudel et al., 2021). Diabetes mellitus patients improve their quality of life with the peer group education approach (Ghasemi et al., 2019)(Krishnamoorthy et al., 2019). Peer group education can be used in diabetes education, this is because the two methods are both able to increase patient understanding of blood sugar and lipid control (Zhao et al., 2019). Peer group education measures can increase knowledge about dementia and have a small but positive impact on the support received from nurses to elderly people with dementia (van Wezel et al., 2020). Peer-assisted learning-based actions were able to increase knowledge of medical students (Brunelli et al., 2020). Peer education measures are effective in reducing non-adherence to taking antiretroviral therapy. This study can be a basis for further studies to be carried out in other areas, because peer education can be influenced by sociodemographic and economic factors (Hussein et al., 2020). Peer education is proven to be able to increase knowledge, understanding, and application of patients in terms of chemotherapy complications they experience (Heydarzadeh et al., 2020). The prevention program using the Peer education strategy the more effective the more effective targeted at youth and youth and provided interactive and action-oriented interventions (Fallace et al., 2019).

Peer group-based technology applications have been developed just like virtual peer support for family can improve wellbeing of their family that have a chronic illness (Jeffrey et al., 2020). One of them is the implementation of a peer support intervention program which is able to reduce the risk of postnatal depression (Shorey et al., 2018). The use of peer group-based text massage can use short messages with an online approach where the results are that the use of online peer groups is more efficient (Swendeman et al., 2019). The use of social media with a 
peer group approach can provide emotional support and increase empowerment in diabetics (Gavrila et al., 2019).

\section{CONCLUSION}

Peer group as an approach Nursing action needs to be done in both hospital and community services. Peer group support and peer group education, both conventional and technology-based, can be applied to patients with breast cancer, diabetes mellitus, HIV AIDS. This can have an impact on the increasingly varied approaches that can be taken by nurses in carrying out nursing actions.

\section{REFERENCES}

Adlard, K. N., Jenkins, D. G., Salisbury, C. E., Bolam, K. A., Gomersall, S. R., Aitken, J. F., Chambers, S. K., Dunn, J. C., Courneya, K. S., \& Skinner, T. L. (2019). Peer support for the maintenance of physical activity and health in cancer survivors: The PEER trial - A study protocol of a randomised controlled trial. BMC Cancer, 19(1), 1-15. https://doi.org/10.1186/s12885-019-5853-4

Ahmadi, Z., Sadeghi, T., \& Loripoor, M. (2018). The outcomes of peer-led diabetes education in comparison to education delivered by health professionals in Iranian patients. Health Education Research, 33(1), 64-72. https://doi.org/10.1093/her/cyx068

Backett-Milburn, K., \& Wilson, S. (2000). Understanding peer education: Insights from a process evaluation. Health Education Research, 15(1), 85-96. https://doi.org/10.1093/her/15.1.85

Brunelli, L., , Annarita Tullio, Giuseppe Perri, L. L., Grillone, L., Giulio Menegazzi, Corrado Pipan, Francesca Valent, S. B., \& MParpinel, A. (2020). Peer education for medical students on health promotion and clinical risk management. January, 1-6. https://doi.org/10.4103/jehp.jehp

Cameron, A. J., Charlton, E., Walsh, A., Hesketh, K., \& Campbell, K. (2019). The influence of the maternal peer group (partner, friends, mothers' group, family) on mothers' attitudes to obesity-related behaviours of their children. BMC Pediatrics, 19(1), 1-8. https://doi.org/10.1186/s12887-019-1726-x

Carvello, M., Zanotti, F., Rubbi, I., Bacchetti, S., Artioli, G., \& Bonacaro, A. (2019). Peersupport: A coping strategy for nurses working at the emergency ambulance service. Acta Biomedica, 90(2), 29-37. https://doi.org/10.23750/abm.v90i11-S.8923

Chime, O. H., Arinze-Onyia, S. U., \& Ossai, E. N. (2019). Examining the effect of peer-support on self-stigma among persons living with hiv/aids. Pan African Medical Journal, 34, 1-10. https://doi.org/10.11604/pamj.2019.34.200.17652

Fallace, P., Aiese, P., Bianco, E., Bolognini, I., Costa, M. P., Esposito, R., Gallé, F., Liguori, G., Pandolfi, R., Pasquarella, C., Savino, G., Valeriani, F., \& Romano Spica, V. (2019). Peer Education strategies for promoting prevention of doping in different populations. Annali Di Igiene, 31(6), 556-575. https://doi.org/10.7416/ai.2019.2316

Gavrila, V., Garrity, A., Hirschfeld, E., Edwards, B., \& Lee, J. M. (2019). Peer Support Through 
a Diabetes Social Media Community. Journal of Diabetes Science and Technology, 13(3), 493-497. https://doi.org/10.1177/1932296818818828

Ghasemi, M., Hosseini, H., \& Sabouhi, F. (2019). Effect of peer group education on the quality of life of elderly individuals with diabetes: A randomized clinical trial. Iranian Journal of Nursing and Midwifery Research, 24(1), 44-49. https://doi.org/10.4103/ijnmr.IJNMR_39_17

Heydarzadeh, L., Alilu, L., Habibzadeh, H., \& Rasouii, J. (2020). The effect of peer education on knowledge, comprehension, and knowledge application of patients regarding chemotherapy complications. Iranian Journal of Nursing and Midwifery Research, 25(1), 40-46. https://doi.org/10.4103/ijnmr.IJNMR_69_19

Hussein, M., Dibaba, B., \& Wondu, Y. (2020). Factors influencing effectiveness of peer education against nonadherence to antiretroviral therapy among adult people living with HIV/AIDS: A pre-post intervention study in Arsi zone, Ethiopia. HIV/AIDS - Research and Palliative Care, 12, 1-7. https://doi.org/10.2147/HIV.S228330

Jeffrey, A., Andracchio, L., Dvorak, M., Lomas, P., Smith, B., \& Borowitz, D. (2020). Virtual Peer Support for People With Cystic Fibrosis and Their Family Members: A Program Evaluation. Journal of Patient Experience, 7(6), 1748-1754. https://doi.org/10.1177/2374373520974322

Kamioka, H. (2019). Preferred reporting items for systematic review and meta-analysis protocols (prisma-p) 2015 statement. Japanese Pharmacology and Therapeutics, 47(8), 1177-1185.

Krishnamoorthy, Y., Sakthivel, M., Sarveswaran, G., \& Eliyas, S. K. (2019). Effectiveness of peer led intervention in improvement of clinical outcomes among diabetes mellitus and hypertension patients - A systematic review and meta-analysis. Primary Care Diabetes, 13(2), 158-169. https://doi.org/10.1016/j.pcd.2018.11.007

McPeake, J., Hirshberg, E. L., Christie, L. M., Drumright, K., Haines, K., Hough, C. L., Meyer, J., Wade, D., Andrews, A., Bakhru, R., Bates, S., Barwise, J. A., Bastarache, J., Beesley, S. J., Boehm, L. M., Brown, S., Clay, A. S., Firshman, P., Greenberg, S., ... Iwashyna, T. J. (2019). Models of Peer Support to Remediate Post-Intensive Care Syndrome. Critical Care Medicine, 47(1), e21-e27. https://doi.org/10.1097/ccm.0000000000003497

Page-Reeves, J., Murray-Krezan, C., Regino, L., Perez, J., Bleecker, M., Perez, D., Wagner, B., Tigert, S., Bearer, E. L., \& Willging, C. E. (2021). A randomized control trial to test a peer support group approach for reducing social isolation and depression among female Mexican immigrants. BMC Public Health, 21(1), 1-18. https://doi.org/10.1186/s12889020-09867-z

Pienaar, M. (2021). A diabetes peer support intervention: Patient experiences using the Mmogomethod ${ }^{\circledR}$. Health SA, 26(Dennis 2003), 1-7.

Poudel, K., Sumi, N., \& Yano, R. (2021). Impact of Peer-Led Cancer Education Program on Knowledge, Health Beliefs, Practice, and Self-Esteem Among Pairs of Nepalese HighSchool Students and Their Knowledge-Sharing Partners. Healthcare, 9(1), 64. https://doi.org/10.3390/healthcare9010064

Pyles, T. E. H., Umi, S. A., Madubuonwu, S., Stiles, A., Devane-Johnson, S., Scott, H., Rhoads, S., \& Russell, J. H. (2021). Breastfeeding Sisters That Are Receiving Support: CommunityBased Peer Support Program Created for and by Women of Color. Breastfeeding Medicine, 16(2), 165-170. https://doi.org/10.1089/bfm.2020.0313 
Rencken, C. A., Harrison, A. D., Mtukushe, B., Bergam, S., Pather, A., Sher, R., Davidson, B. J., Carrihill, M., Matiwane, M., Kuo, C., Galárraga, O., \& Hoare, J. (2021). "Those People Motivate and Inspire Me to Take My Treatment." Peer Support for Adolescents Living With HIV in Cape Town, South Africa. Journal of the International Association of Providers of AIDS Care, 20, 1-9. https://doi.org/10.1177/23259582211000525

Richards et al. (2018). 乳鼠心肌提取 HHS Public Access. Physiology \& Behavior, 176(5), 139 148. https://doi.org/10.1016/j.pedn.2018.12.011.Evaluation

Scherzer, C. R., Ranney, M. L., Jain, S., Bommaraju, S. P., Patena, J., Langdon, K., Nimaja, E., Jennings, E., Francesca, L., \& Floor, S. (2020). HHS Public Access. 5(1), 1-14. https://doi.org/10.20900/jpbs.20200001.Mobile

Shannalee R. Martinez, Maresha S. Gay, and L. Z. (2016). 乳鼠心肌提取 HHS Public Access. Physiology \& Behavior, 176(1), 139-148. https://doi.org/10.1177/0145721719844547.Effectiveness

Shorey, S., Chee, C., Chong, Y. S., Ng, E. D., Lau, Y., \& Dennis, C. L. (2018). Evaluation of technology-based peer support intervention program for preventing postnatal depression: Protocol for a randomized controlled trial. Journal of Medical Internet Research, 20(3). https://doi.org/10.2196/resprot.9416

Singstad, M. T., Wallander, J. L., Greger, H. K., Lydersen, S., \& Kayed, N. S. (2021). Perceived social support and quality of life among adolescents in residential youth care: a crosssectional study. Health and Quality of Life Outcomes, 19(1), 1-12. https://doi.org/10.1186/s12955-021-01676-1

Swendeman, D., Arnold, E. M., Harris, D., Fournier, J., Comulada, W. S., Reback, C., Koussa, M., Ocasio, M., Lee, S. J., Kozina, L., Fernández, M. I., \& Rotheram, M. J. (2019). Textmessaging, online peer support group, and coaching strategies to optimize the HIV prevention continuum for youth: Protocol for a randomized controlled trial. JMIR Research Protocols, 8(8). https://doi.org/10.2196/11165

Toija, A. S., Kettunen, T. H., Leidenius, M. H. K., Vainiola, T. H. K., \& Roine, R. P. A. (2019). Effectiveness of peer support on health-related quality of life in recently diagnosed breast cancer patients: a randomized controlled trial. Supportive Care in Cancer, 27(1), 123-130. https://doi.org/10.1007/s00520-018-4499-0

van Wezel, N., van der Heide, I., Devillé, W. L., Kayan Acun, E., Meerveld, J. H. C. M., Spreeuwenberg, P., Blom, M. M., \& Francke, A. L. (2020). Effects of an educational peergroup intervention on knowledge about dementia among family caregivers with a Turkish or Moroccan immigrant background: A cluster randomised controlled trial. Patient Education and Counseling, xxxx. https://doi.org/10.1016/j.pec.2020.11.008

Warshaw, H., \& Edelman, D. (2019). Building Bridges Through Collaboration and Consensus: Expanding Awareness and Use of Peer Support and Peer Support Communities Among People With Diabetes, Caregivers, and Health Care Providers. Journal of Diabetes Science and Technology, 13(2), 206-212. https://doi.org/10.1177/1932296818807689

Williams, E. M., Egede, L., Oates, J. C., Dismuke, C. L., Ramakrishnan, V., Faith, T. D., Johnson, H., \& Rose, J. (2019). Peer approaches to self-management (PALS): Comparing a peer mentoring approach for disease self-management in African American women with lupus with a social support control: Study protocol for a randomized controlled trial. Trials, 20(1), 1-13. https://doi.org/10.1186/s13063-019-3580-4 
Journal of Advanced Multidisciplinary Research

Vol. 2, No. 1, 2021, pp. 12-20

ISSN: 2723-6978

http://jurnal.unissula.ac.id/index.php/JAMR DOI: http://dx.doi.org/10.30659/JAMR.2.1.12-20

Zhao, X., Yu, X., \& Zhang, X. (2019). The Role of Peer Support Education Model in Management of Glucose and Lipid Levels in Patients with Type 2 Diabetes Mellitus in Chinese Adults. Journal of Diabetes Research, 2019. https://doi.org/10.1155/2019/5634030. 